\title{
ENVEJECIMIENTO DOMESTICO, CORRESIDENCIA Y OFERTA LABORAL DE HOMBRES Y MUJERES
}

\author{
DOMESTIC AGING, CO-RESIDENCE AND LABOR SUPPLY OF MEN \\ AND WOMEN
}

\author{
JORGE A. PAZ* \\ CONICET-IELDE/UNSa
}

\begin{abstract}
This paper explores the correlation between the presence and the number of elderly people in households and the labor supply of men and women between 18 and 54 years old, using data from the Argentina Permanent Household Survey for an extended period. Does the presence of elderly people alter the labor participation of men and women at central ages? It is proposed that this is so and that the correlation direction correlation will depend on the contribution they make to the home, or the demand for care they generate. Through the application of traditional multivariate models, it was found that the presence and the number of elderly people in the household is negatively related to the labor participation of men and women at central ages, and, in some cases, it relates positively with the participation of women. The effects on working hours are more elusive.
\end{abstract}

Keyword: Ageing, fertility, labor force participation, Argentina, elderly people, labor supply, time use, gender economics, feminist economics, economics of care.

JEL Classification: J14, J22, O54.

\footnotetext{
1 Investigador Independiente del Consejo Nacional de Investigaciones Científicas y Técnicas (CONICET) con sede en el Instituto de Estudios Laborales y del Desarrollo Económico (IELDE), Universidad Nacional de Salta, Argentina. Este trabajo fue financiado parcialmente por el Consejo de Investigación de la Universidad Nacional de Salta, Proyecto 2509/0. El autor agradece los comentarios recibidos de árbitros anónimos que contribuyeron a mejorar sensiblemente la calidad del artículo. Se agradece también las observaciones realizadas por los investigadores del departamento de Economía de la Universidad de San Andrés, Argentina, en el seminario realizado el 28 de agosto de 2018. Los errores que persisten son responsabilidad del autor.
} 


\section{Resumen}

En este trabajo se explora la correlación entre la presencia y la cantidad de personas mayores en los hogares y la oferta laboral de hombres y mujeres entre 18 y 54 años, usando datos de la Encuesta Permanente de Hogares de Argentina para un extenso período. ¿La presencia de personas mayores altera la participación laboral de hombres y mujeres en edades centrales? Se plantea que esto es así y que la dirección de la correlación dependerá del aporte que ellas hagan al hogar, o de la demanda de cuidado que generen. Mediante la aplicación de modelos multivariados tradicionales se encontró que la presencia y la cantidad de personas mayores en el hogar se relaciona negativamente con la participación laboral de hombres y mujeres en edades centrales, y, en algunos casos, positivamente con la participación de las mujeres. Los efectos sobre las horas de trabajo son más elusivos.

Palabras clave: Envejecimiento, fecundidad, participación económica, Argentina, personas mayores, oferta laboral, economía del cuidado, economía feminista, usos del tiempo, economía del género.

Clasificación JEL: J14, J22, O54.

\section{INTRODUCCION}

Recientemente se ha revitalizado el debate acerca de los determinantes de la participación de la mujer en el mercado de trabajo, al menos en algunos países de América Latina (Beccaria et al., 2017). Probablemente tuvo que ver con esto el claro estancamiento de la tasa de actividad femenina observado durante la última década. Gasparini y Marchionni (2015) muestran que, en América Latina durante el período 1992-2002, la tasa de actividad femenina aumentaba a razón de 9 puntos porcentuales (pp) por año, mientras que en la década 2002-2012, lo hizo a razón de 3 pp por año. Dicho estancamiento no se dio sólo en los países de la región sino también en otras economías con diferente nivel de desarrollo relativo (Health y Jayachandran, 2017; Klasen y Pieters, 2015).

Los determinantes tradicionalmente analizados de la participación femenina son: la fecundidad (Bloom et al., 2009), el nivel educativo (Health y Jayachandran, 2017; Psacharopoulos y Thanatos, 1989), y, en varios estudios, la situación del mercado laboral masculino por la probable ocurrencia ya sea del llamado "efecto del trabajador adicional", o del "efecto del trabajador alentado/desanimado" (Paz, 2007). A diferencia de los dos primeros, este último tiene un carácter más bien coyuntural, por lo que podría excluirse de la lista si lo que se quiere es entender un quiebre estructural de una tendencia de mediano o largo plazo, como la descrita por la tasa de actividad 
femenina. Este ha sido el tratamiento dado al tema en una extensa literatura cuyo origen puede situarse en la década de 1970 a raíz de la expansión de la tasa de actividad de las mujeres en los países de mayor nivel de desarrollo que se dio concomitante a un fuerte descenso del número promedio de hijos tenidos por las mujeres y a la expansión educativa (ver, por ejemplo, Killingsworth y Heckman, 1986).

A pesar de su amplia aceptación, los determinantes mencionados no son satisfactorios para dar cuenta del estancamiento de la tasa de actividad de las mujeres y de la suave caída de la tasa de actividad de los hombres, referidas en el primer párrafo. La fecundidad siguió descendiendo, alcanzando en algunos países niveles muy bajos, y la educación sigue aumentando inexorablemente (Barro \& Lee, 2016). Así, descartados estos determinantes como claves para dar cuenta de la evolución de las tasas de actividad, hay que explorar otros, algunos de los cuales recibieron poca atención en la literatura. Puede suceder, por ejemplo, que las mujeres más propensas a participar ya estén en el mercado de trabajo y que permanecen en la inactividad aquellas que: a) creen (ellas y sus parejas) que la mujer debe encargarse de los quehaceres domésticos y de las tareas de cuidado (Paz, 2018); o bien, b) ocupan su tiempo en dichas tareas por diversos motivos, uno de los cuales es a). Esta última hipótesis incluye la especialización al interior de los hogares: las mujeres en tareas domésticas y de cuidado y los hombres en trabajos para el mercado. Asimismo, de b) queda un punto por develar: ¿cuidar a quién? Justamente esta hipótesis es la contracara de aquella que se usó para entender el aumento de la tasa de actividad: la fecundidad descendió, la demanda de cuidado cayó, liberando tiempo disponible para dedicarlo al trabajo remunerado. Si cada vez hay menos niños para cuidar, entonces ¿por qué la participación se estancó?; o bien, ¿por qué cierto grupo de mujeres (como las escandinavas) tienen cada vez más hijos y no declinan su participación en el mercado?

Las respuestas a estos interrogantes remiten a los puntos a) y b) nuevamente, porque: (i) el tiempo de cuidado y de trabajo doméstico se distribuye desigualitariamente entre hombres y mujeres, (ii) si bien hay cada vez menos niños, el envejecimiento demográfico está provocando un aumento de la demanda de cuidado, no ya de niñas y niños, sino de personas mayores. Si se cumple ii), dicha demanda recae mayoritariamente sobre las mujeres. Pero para que esto ocurra debe darse que las personas mayores cada vez sean más, que vivan más años, y que requieran cuidado, que dicho cuidado no esté cubierto por alguien cercano (cónyuge) o por profesionales (servicios formales). Esto es una condición necesaria, dado que si las personas mayores están en buenas condiciones de salud podrían generar "transferencias hacia abajo" en términos generacionales, colaborando con recursos monetarios y no monetarios, liberando tiempo de adultas/os en edades centrales que podría ser utilizado para el trabajo para el mercado.

Este artículo se ocupa de la relación entre la presencia y cantidad de personas mayores y la participación económica en hogares con corresidencia intergeneracional; esto es hogares compuestos por personas en edades centrales (18-54) y personas mayores (65 y más) con o sin niñas y niños (0-17). El fenómeno adquiere relevancia precisamente por el panorama demográfico de Argentina, un país cerca de concluir 
la primera transición demográfica y en los que comienzan a visualizarse signos de la segunda, según se verá enseguida. Se analiza entonces el efecto que sobre la participación laboral de hombres y mujeres en edades centrales provoca la presencia de niñas y niños como rasgo destacado de la primera transición (supuestamente ya concluida), y de personas mayores en el hogar en el que residen, como rasgo destacado de la segunda transición (en proceso). Este último aspecto ha recibido muy poco tratamiento en la literatura especializada.

El trabajo está organizado según el siguiente plan: en la próxima sección se describe el marco conceptual sobre el que se apoya la investigación realizada. La sección IIII contiene una revisión de la literatura relevante, y en la IV se describen los datos usados y la metodología implementada para responder a los principales interrogantes planteados en el documento. En la sección V se discuten los resultados alcanzados y se enumeran al final (sección VI) las conclusiones a las que se arribó en este artículo.

\section{MARCO CONCEPTUAL}

Los primeros trabajos que tomaron en cuenta la demanda de cuidado de personas mayores y sus efectos sobre la oferta laboral de las personas en edades centrales fueron Wolf y Soldo (1994), y Ettner (1995 y 1996). A diferencia de estos, interesados exclusivamente en la demanda de cuidado, en este trabajo se plantea que la corresidencia intergeneracional podría generar efectos de dos tipos sobre la participación económica y el esfuerzo laboral: uno positivo y otro negativo. El primero se daría si las personas mayores corresidentes: a) colaboran con los quehaceres del hogar, tanto en tareas domésticas como con el cuidado de otros miembros (por ejemplo, de niñas/os); y/o b) aportan ingresos no laborales (provenientes de fuentes tales como jubilación/pensión), o servicios de residencia (propiamente, vivienda), disminuyendo la necesidad de obtener recursos de los miembros del hogar en edades centrales. En términos microeconómicos, esto último implicaría un efecto ingreso, que podría reducir la oferta de trabajo de las personas adultas en edades centrales, aumentando su salario de reserva.

El efecto negativo se daría si las personas mayores presentes en el hogar requieren cuidado de los miembros en edad central. Esto podría darse en contextos económicos, sociales y sanitarios diversos. Por ejemplo, si las personas mayores no cuentan con recursos económicos propios, si no tienen pareja para compartir los esfuerzos que requieren los quehaceres domésticos y/o si se encuentran en un estado de salud que demande cuidados específicos, que esos servicios de cuidado no sean provistos por profesionales (o, al menos, no en la cantidad necesaria), y que los cuidados, además, no sean distribuidos de manera igualitaria entre todos los potenciales cuidadores.

Los tres factores mencionados, dos positivos y uno negativo, implican transferencias que se producen entre los miembros del hogar, las que se diferencian entre sí por su dirección, tomando en cuenta ambas generaciones: hacia abajo, en la medida en que 
sean las personas mayores las que colaboran con la actividad de las/os adultas/os en edades centrales; hacia arriba, en el caso que sean las/os adultas/os en edades centrales quienes se ocupen del cuidado de las personas mayores. En el primer caso puede decirse que estas últimas tienen un rol activo en las tareas de reproducción, mientras que, en el segundo, un rol pasivo. Según cuál sea el tipo de transferencias que predomine, el efecto sobre la participación económica será positivo, negativo o neutro. Es decir, no existe una razón teórica que permita adelantar cuál será el resultado final de la presencia de personas mayores sobre la oferta laboral de adultas/os en edades centrales en los hogares con corresidencia intergeneracional. Lo antedicho se resuelve con la evidencia empírica y, como tal, está sujeto a variables que trascienden lo puramente económico, como costumbres, formas de arreglos residenciales preexistentes, entre tantas otras.

A nivel agregado, el efecto total será el resultado del balance de estas fuerzas, y, por lo tanto, el resultado final no puede ser predicho por un esquema teórico. Hasta podría suceder que el efecto fuese nulo, lo que ocurriría si las fuerzas que hacen aumentar la participación se compensan con aquellas que la hacen reducir. Llegados a este punto viene bien aclarar que por oferta laboral se entiende tanto la decisión de estar o no estar en el mercado de trabajo, esto es, la decisión de participación, como las horas dedicadas a la generación de ingresos. Este estudio se ocupará de ambas, considerando tanto la participación laboral como el número de horas ofrecidas al trabajo para el mercado.

Existen varios motivos para sospechar que el efecto final de la corresidencia sobre la oferta laboral de adultas/os en edades centrales (positivo, negativo o neutro) difiera entre grupos de individuos. Por ejemplo, puede suceder que la reducción de horas dedicadas al trabajo remunerado sea mayor entre las mujeres que entre los hombres, debido a la división intrahogar del trabajo doméstico no remunerado. Puede ocurrir también que el costo de cuidado en términos de horas de trabajo para el mercado sea mayor para los estratos de ingresos familiares bajos que no pueden acceder a servicios de atención pagados.

Desde la perspectiva de la oferta de trabajo y, en consecuencia, del crecimiento económico, el saber cuál de ambos efectos predomina es muy importante. Si la corresidencia genera transferencias hacia abajo, el envejecimiento tendrá efectos positivos sobre de la oferta de trabajo y el crecimiento económico. Por el contrario, si la corresidencia implica transferencias hacia arriba, tendrá efectos negativos sobre de la oferta de trabajo y el crecimiento económico. Además, si la corresidencia es diferencial por estrato socioeconómico o de clase, el efecto tendrá impactos distributivos que valdría la pena conocer, pero que no serán abordados en este documento.

\section{LITERATURA PERTINENTE}

Las investigaciones sobre los determinantes de la participación laboral se han centrado principalmente en la población femenina y, dentro de este grupo, en las 
casadas entre 25 y 54 años (Killingsworth y Heckman, 1986; Blau y Khan, 1996). Es precisamente este grupo el que explica la gran expansión de la participación de la mujer en la actividad económica remunerada, en los países desarrollados y en algunos en desarrollo (Pieter y Klasen, 2011).

En esos análisis jugó un rol protagónico el tiempo dedicado a la crianza y al cuidado de los hijos, tanto en la decisión de estar en el mercado de trabajo como en el número de horas dedicadas a un empleo remunerado. En los estudios que abordan este tema en América Latina y el Caribe se ha venido evaluando la posibilidad de que la caída de la fecundidad pueda contribuir al crecimiento económico y a la reducción de la desigualdad y la pobreza, a través de lo que se ha dado en llamar el "bono de género" (Martínez et al., 2013; Pagés y Piras, 2010).

Menos numerosos son los aportes que abordan la relación entre la corresidencia intergeneracional y la participación económica de adultos en edades centrales. Este no es un hecho menor en un país como Argentina, con muy baja fecundidad, una población envejecida para el patrón regional, y con una prolongada esperanza de vida a diferentes edades (ver sección III). Aparece así en escena nuevamente el tema de cuidado. La reducción de la demanda de cuidado que habría provocado la primera fase de transición demográfica con la caída de la fecundidad podría aumentar en la segunda transición, debido al envejecimiento de la población y a la creciente demanda de servicios de cuidado que ello trae aparejado.

Los trabajos de Wolf y Soldo (1994) y de Susan Ettner (1995 y 1996) son los primeros que se ocuparon detalladamente de este importante tema. Estos estudios aparecen a mediados de los 90, cuando el fenómeno del envejecimiento estaba plenamente instalado en EE.UU. Los resultados que obtienen estos autores no son concordantes. Mientras que los primeros no encuentran efectos de la presencia de personas mayores en el hogar sobre la oferta de trabajo, Ettner (1995 y 1996) afirma que la reduce, y que dicho impacto no solo es significativo en las horas trabajadas, sino que saca personas (especialmente mujeres) del mercado laboral, y que eso está relacionado con el estado de salud de las personas mayores.

A resultados similares a los de Ettner llegan también otros autores (Heitmueller, 2004; Leigh, 2010 y Von Houtven et al., 2013), usando siempre el concepto de "cuidado informal" entendiendo por tal aquella atención realizada por familiares y/o vecinos a personas con discapacidad crónica, principalmente personas mayores (Carmichael, 2010).

Excepto el trabajo de Crespo (2006) que encuentra evidencias similares para países de Europa, después del trabajo de Ettner (1995), la atención principal de este tipo de estudios se orientó a la demanda de cuidado más que a la corresidencia propiamente dicha; y si bien ambos fenómenos están conectados, no significan lo mismo, como se dijo antes. Una persona puede hacerse cargo del cuidado de una persona mayor sin corresidir y esa "invisibilidad" del cuidado podría estar subestimando el efecto medido sobre la oferta de trabajo de las personas adultas en edades centrales. 
Las investigaciones más recientes acerca de los efectos de la corresidencia intergeneracional sobre la participación laboral de la población adulta en edades centrales abordaron el problema analizando la demanda de cuidado de personas con discapacidad (por ejemplo, Maurer-Fazio et al., 2009 y Shen y Chen, 2012). Es de destacar que estas investigaciones provienen en particular de Asia, donde no solo se ha observado un envejecimiento demográfico acelerado en los últimos decenios y una particular evolución de la participación de la mujer en la actividad económica, sino que se han lanzado programas (como el de seguros de largo plazo analizado por Sugawara y Nakamura, 2014) o el programa de salud estudiado por Liu (2010) por ejemplo) que impactaron claramente en los mercados de servicios de cuidado. Quizá provocado por estos programas, los resultados encontrados por estos autores muestran efectos positivos de la demanda de cuidado sobre la participación económica.

En América Latina es el estudio de Bravo y Puentes (2012) el que analiza el problema de la participación laboral de mujeres que corresiden con personas mayores. Estos autores encuentran un efecto negativo y significativo sobre la participación, luego de controlar el impacto por un conjunto de variables independientes. Lo curioso es que dicho efecto desaparece cuando los autores corrigen sus estimaciones por endogeneidad. Claro está que juegan un papel importante en todos los casos los instrumentos elegidos para corregir por endogeneidad y estos no dependen de las necesidades del analista sino de las variables disponibles en las bases de datos usadas.

Otros autores no detectan relación fuerte entre ambos fenómenos. En este sentido, resulta particularmente interesante el trabajo de Meng (2009) para Alemania porque los datos de panel que utiliza le permiten controlar la heterogeneidad no observada. En el ejercicio realizado encuentra efecto negativo sobre la oferta laboral, más bajo para las mujeres que para los hombres. Esto llama la atención porque como se advierte, en Alemania existe una preferencia de la gente a realizar los cuidados en el domicilio y que sean realizados por familiares. Tampoco Unger (2013) encuentra relación para Noruega, Suecia y Dinamarca, aunque hay un efecto para aquellos cuidadores informales que ocupan más de 30 horas semanales con las personas mayores corresidentes.

Por último, el estudio de Posadas y Vidal-Fernández (2012) se aparta claramente del resto, porque ellas examinan el rol de personas mayores en el cuidado de niños en el hogar, y el efecto que esa situación ejerce sobre la participación económica de las mujeres. Además, las autoras exploran las implicancias distributivas de este fenómeno. En sus estimaciones (realizadas con variables instrumentales) encuentran un efecto positivo y fuerte sobre la participación económica, que puede adjudicarse a la ayuda proporcionada por las personas mayores.

Entre los estudios examinados hay diferencias en las fuentes de datos utilizadas y principalmente en los métodos de trabajo, a pesar de tratarse todos ellos de un abordaje cuantitativo al problema. Los estudios que aplican variables instrumentales utilizan instrumentos diversos, aunque todos parten del trabajo de Ettner (1995), quien afirma la necesidad de corregir endogeneidad usando variables proxies del estado de salud de los padres, su educación, y el estrato socioeconómico de origen. También son 
relevantes los aportes de corresidencia como el de Guzmán y Hakkert (2001), quienes estiman funciones de corresidencia y usan como variables explicativas el género de las personas mayores y el estrato socioeconómico del hogar.

Por ejemplo, Bravo y Puentes (2012) usan como instrumentos el número de personas mayores de 15 años (hijas e hijos de las mujeres) presentes en el hogar, basándose en la hipótesis del aumento en el número de cuidadores potenciales que estaría relacionado con la atención de las personas mayores, pero no con la participación de la mujer en el mercado de trabajo. Estos autores mencionan como instrumentos importantes la edad de los tres amigos más cercanos, la edad y la salud de los padres y el número de hermanos de la persona cuya participación está siendo evaluada.

En suma, los instrumentos alrededor de los cuales giran todos los trabajos analizados son: educación de los padres, empleo y características de los hermanos no residentes, vivienda propia, servicios de electricidad en la vivienda, y condición de actividad de madres y padres (Magnani y Rammoham, 2006). Otros (Liu, 2010 por ejemplo) trabajan con variables proxies de necesidad de atención de madres y padres y la corresidencia con otros miembros de la familiar que pueden colaborar en las tareas de cuidado. Un aporte interesante lo proporcionan Maurer-Fazio et al. (2009), que incluyen como instrumentos el porcentaje de hogares corresidentes en el distrito, edad de las personas mayores y variables que permiten captar interacciones.

\section{DATOS Y ESTRATEGIA METODOLOGICA}

\section{A. Origen de los datos}

Se usaron datos de la Encuesta Permanente de Hogares (EPH), relevamiento realizado por el Instituto Nacional de Estadística y Censos (INDEC), con la colaboración de las direcciones provinciales de estadística. La elección del período cubierto por el análisis, el comprendido entre los años 2004 y 2018, se corresponde con la denominada EPH-Continua (EPHC) y que contiene información homogénea proveniente de un cuestionario que ha permanecido sin cambios. No obstante, hubo en el período algunos problemas, quizá el más serio fue la intervención del INDEC ocurrida entre 2007 y 2015 y que afectó claramente algunos programas del Instituto, más claramente los relacionados con precios.

La EPH se aplica en Argentina desde el año 1973 a través de la medición puntual de dos ondas anuales (mayo y octubre). A partir de 2003, la EPH pasó a ser un relevamiento continuo (EPHC) que produce datos con frecuencia trimestral. La muestra en la que se basa es probabilística, estratificada, en dos etapas de selección, está distribuida a lo largo del período respecto del cual se brinda información (el trimestre) y el relevamiento se desarrolla durante todo el año.

Con una muestra trimestral típica es de un poco más de 26 mil viviendas, la EPH produce estimaciones trimestrales válidas para un conjunto de los 31 aglomerados 
urbanos (también llamados “centros urbanos”) agrupados en seis regiones estadísticas: Gran Buenos Aires, Nordeste, Noroeste, Centro y Sur (o Patagonia).

La oferta laboral es captada a través de la participación económica y de las horas semanales dedicadas al trabajo remunerado. La edad de las personas mayores es usada como un proxy de su estado de salud, y el sexo con la división del trabajo doméstico ${ }^{1}$. Las variables que se correlacionan como controles son la edad, la educación, la cantidad de niñas y niños, y la región de residencia. Una primera revisión de los datos usados puede hacerse con la información resumida en la Tabla A.1 del Apéndice de Tablas. Se muestran ahí los valores medios de cada una de las variables para el año 2018 y en el análisis descriptivo se destacan algunas de ellas para el período completo 2004-2018.

Para las estimaciones se considerará "población adulta en edades centrales" a hombres y mujeres con edades comprendidas entre los 18 y los 54 años. Se excluyen a menores de 17 años, muchas/os de las/os cuales todavía están cursando su escolaridad secundaria, por lo que la opción laboral no se considera la central, menos aún en Argentina, donde la escolaridad está muy extendida. También se dejan fuera de la población de personas en edad central a las de 55 y más años. Ya que se supone que el período fértil comienza a los 15 años, hay razones para creer que existe una distancia generacional entre las personas mayores más jóvenes (65 años) y las personas adultas en edades centrales más viejas (55 años): 10 años.

Además, el estudio se concentró en aquellos hogares en los que se encontraron personas mayores ${ }^{2}$. Es decir, que las estimaciones cubren hogares de diverso tipo y solo excluye los unipersonales.

\section{B. Estrategia metodológica}

Se supone aquí que la participación en el mercado de trabajo de hombres y mujeres, y el tiempo dedicado por ellas/os a un empleo remunerado, están relacionados de una manera u otra con un conjunto de variables observables, algunas de las cuales pueden estar determinadas conjuntamente con la decisión de participación económica propiamente dicha o con las horas dedicadas al trabajo por un pago. Las variables observables son las que se encuentran disponibles en la fuente de datos que se utiliza en este estudio: la EPH.

La decisión de participar en el mercado laboral puede ser analizada estimando el siguiente modelo:

$$
z_{i}^{*}=\beta y_{i}+\Gamma X+u_{i}
$$

1 Hay abundante literatura acerca de la distribución de las tareas domésticas en el hogar. Las mujeres dedican una parte mayor de su jornada a la atención de tareas domésticas no remuneradas. Un resumen estadístico de esta evidencia puede consultarse en https://oig.cepal.org/es/indicadores/tiempo-total-trabajo.

2 Esto surgió como consecuencia de una sugerencia de uno de los revisores externos de este artículo. 
Donde $z_{i}^{*}$ es una variable latente, no observable, que representa la probabilidad de participación del individuo "i" en la actividad económica. Lo que se puede observar con los datos disponibles, es una variable dicotómica $\left(z_{i}\right)$ que toma valor "0" si el individuo está fuera del mercado laboral, y "1" si participa. En términos simbólicos esto puede escribirse como sigue:

$$
z_{i}=\left\{\begin{array}{ccc}
1 & \text { si } & z_{i}^{*}>0 \\
0 & \text { si } & z_{i}^{*} \leq 0
\end{array}\right.
$$

En este caso $y_{i}$ en [1] es la estructura por edad y sexo de las personas mayores en el hogar en el que reside la persona adulta en edad central $i$. Por su parte, $X$ es una matriz que incluye variables observables que influyen en la participación económica de la población y en la oferta de horas de trabajo remunerado, ampliamente reconocidas en la literatura (Killingsworth \& Heckman, 1986). De ese conjunto se consideraron aquí las variables que pudieron observarse en la EPH: edad, nivel educativo, presencia de niñas y niños y región de residencia.

De la misma manera, el tiempo que una persona dedica a trabajar por una remuneración puede ser especificado de manera idéntica:

$$
h_{i}=\gamma y_{i}+\Xi X+\varepsilon_{i}
$$

En [1] y en [2] aparecen además los términos $\beta, \Gamma, \gamma$ y $\Xi$, que simbolizan los parámetros a estimar. Los parámetros $\beta$ y $\gamma$ resumen la correlación entre la presencia y cantidad de personas mayores y la oferta de trabajo de hombres y mujeres en edades centrales (20-49) que residen en hogares con personas mayores. La oferta laboral medida como participación y el esfuerzo (horas), respectivamente.

La significatividad estadística de dichos parámetros no permitiría rechazar la ausencia de correlación, con lo cual podría decirse que la presencia de personas mayores no tiene que ver con la participación económica de las personas adultas en edades centrales corresidentes. Si el parámetro resultase significativo, habría que analizar el signo que arroja: el signo negativo implicaría que la presencia de personas mayores en el hogar está asociado a una participación económica o a un esfuerzo laboral menor de los adultos en edades centrales. Si es positivo podría pensarse que la presencia de personas mayores favorece la participación de adultos centrales.

Por su parte, $\Gamma$ y $\Xi$ son vectores que simbolizan los parámetros de las variables de control, cuya significancia y signo son los que aparecen en la literatura de participación económica y que están sustentados por la teoría de la participación económica de la población. Igualmente, más abajo se proporcionan pistas acerca del signo esperado para cada una de las variables incluidas en la matriz $X$. 
Para estimar [1] se usó un modelo probit y para estimar [3], un modelo tobit, debido a la truncación a la izquierda que presenta la variable dependiente (horas trabajadas).

\section{RESULTADOS}

\section{A. Repaso descriptivo}

La participación económica de la población no puede ser adecuadamente analizada sin tener en cuenta tanto el diferencial por género como por edad. En el Gráfico 1 puede apreciarse la evolución de la tasa de actividad, principal indicador del nivel de participación. Se observa claramente un aumento entre las mujeres y una reducción un tanto suave entre los hombres. La verdadera expansión del nivel de participación económica femenina se dio durante las décadas de 1980 y 1990 para luego estancarse desde comienzos del presente siglo hasta la fecha (año 2019).

En los países más desarrollados como EE.UU. y los de Europa Occidental puede verse una imagen similar a la anterior, aunque la expansión registrada en Argentina durante los 80 comienza en ellos una década antes, durante los años 70. Lo que resulta perfectamente coincidente es el estancamiento a partir de los 2000.

\section{GRAFICO 1}

TASAS DE ACTIVIDAD POR SEXO, ARGENTINA, 1974-2019

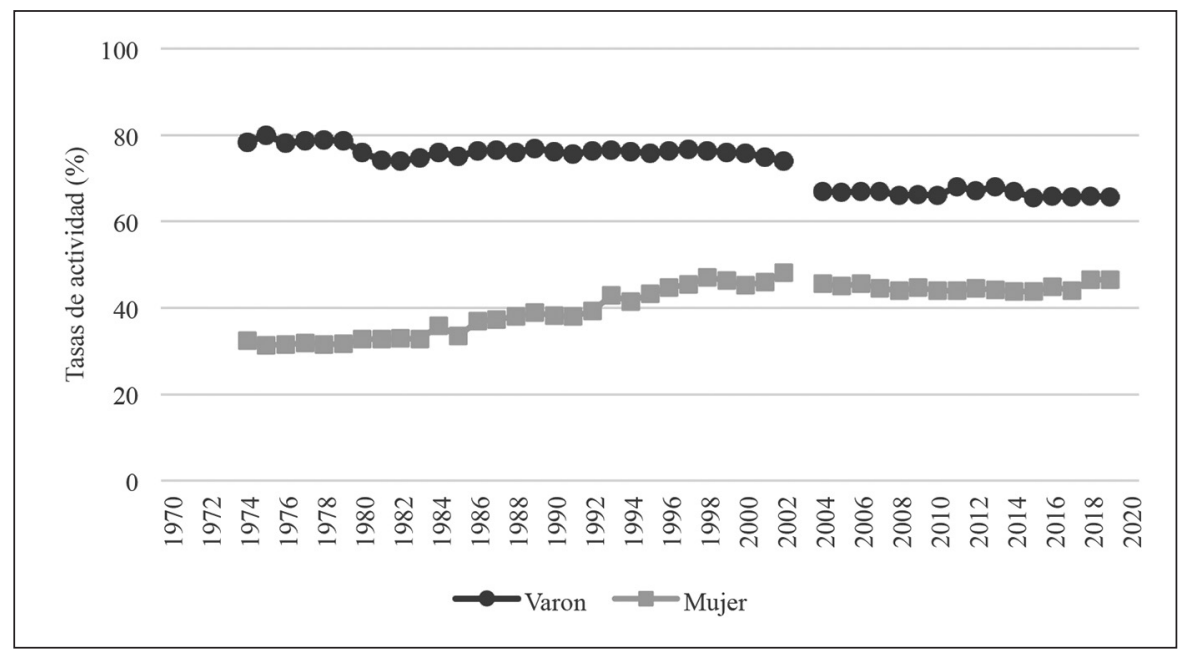

Nota: El corte en 2003 obedece a falta de información por cambio de modalidad (ver texto).

Fuente: Elaboración propia con datos de INDEC, EPH. 
La tasa de actividad masculina no arroja una atendencia tan clara como la femenina. La caída que se produce luego del corte en el año 2003 obedece en buena medida al cambio de la metodología de relevamiento de la Encuesta Permanente de Hogares (EPH), la fuente de la cual proviene la información contenida en el Gráfico 1. La principal conclusión que se obtiene de dicho Gráfico es que la brecha entre hombres y mujeres persiste y que no hay evidencias de que pueda cerrarse, al menos en el mediano plazo. Además, dicha diferencia se verifica, como lo muestran los datos resumidos en el Gráfico 2, en todos los grupos de edad, siendo mayor en aquellos de edades más avanzadas.

Esto último es lógico, en la medida en que esta población proviene de generaciones en las que la disparidad por género era mucho más amplia y a que el arrastre de los patrones de participación suele ser muy marcado, como lo muestran los estudios que abordan este tema (por ejemplo, Klasen y Pieters, 2015).

Los cambios en el mercado de trabajo y en los patrones de participación fueron acompañados de otros fenómenos relacionados, como la expansión educativa (Lee \& Lee, 2016) y las transformaciones demográficas ocurridas a lo largo del tiempo. De todos estos cambios interesan particularmente en este artículo aquellos que tienen que ver con los cambios demográficos, especialmente los vinculados a la fecundidad y el envejecimiento de la población.

\section{GRAFICO 2}

TASAS DE ACTIVIDAD POR SEXO Y EDAD. ARGENTINA, 2019

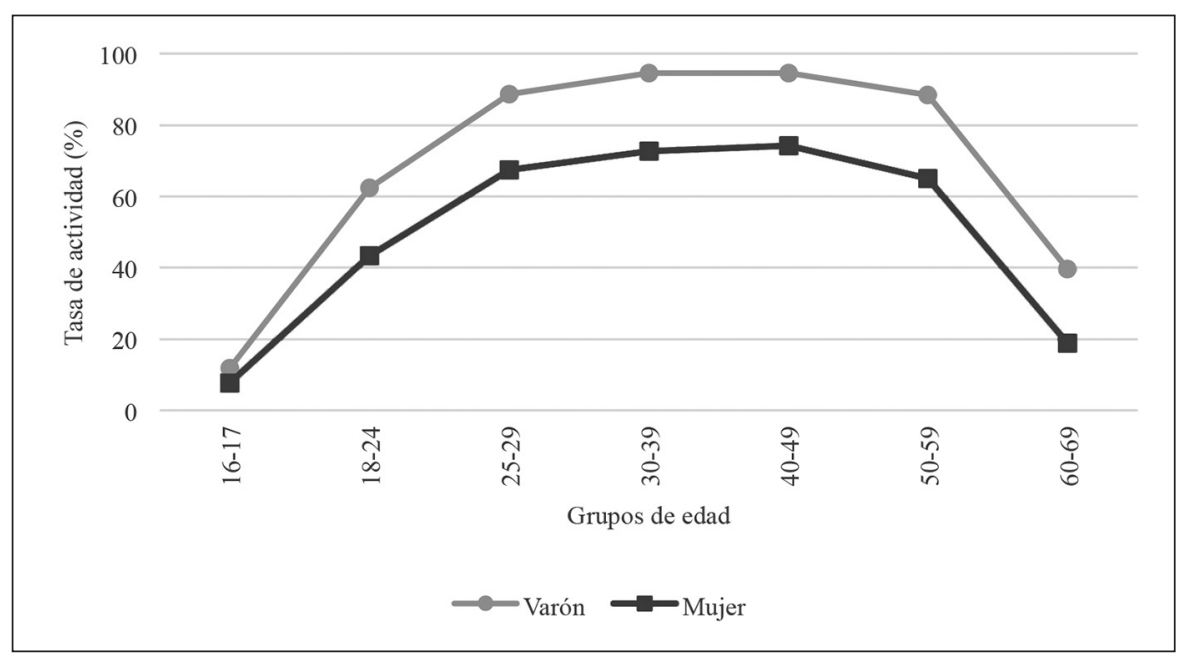

Fuente: Elaboración propia con datos de INDEC, EPH. 
Argentina se ubica entre los países de América Latina con más bajos niveles de fecundidad. Pero lo que caracteriza más y mejor al país en este sentido es su evolución a lo largo del tiempo. Argentina comenzó el siglo pasado con niveles de fecundidad similares a los registrados en los países de Europa Occidental en ese momento, aunque luego su evolución no siguió la velocidad de descenso que se verificó en aquellos. Igualmente, como puede apreciarse en el Gráfico 3, la brecha permanece en los niveles observados en el quinquenio 1950-55, lo cual obedece al comportamiento de determinados grupos de edad, como se verá en seguida.

Entre los quinquenios 1975-1980 y 2015-2020 la fecundidad cayó más en los grupos de edades centrales (Gráfico 4), un patrón que suele conectarse con la evolución de la participación económica. Killingsworth \& Heckman (1986) muestran que el aumento de la tasa de actividad de las mujeres en EE.UU. se explica en buena medida por la caída de la fecundidad entre las casadas, las que se agrupan, mayoritariamente, entre los 25 y 34 años (Montgomery \& Trussell, 1986).

Hacia 1975-80 la edad modal de la fecundidad se ubicaba en el grupo de 25-29, mientras que en la fecha más reciente se observa una meseta entre los 20-34 años. El cambio en el soporte de la distribución se reflejó una caída en la edad media de la fecundidad. Claramente se trata de un momento del ciclo de vida en que es altamente probable que se haya consolidado la inserción en el mercado de trabajo.

\section{GRAFICO 3}

TASAS DE FECUNDIDAD, ARGENTINA Y EUROPA OCCIDENTAL, 1950-55 A 2015-20

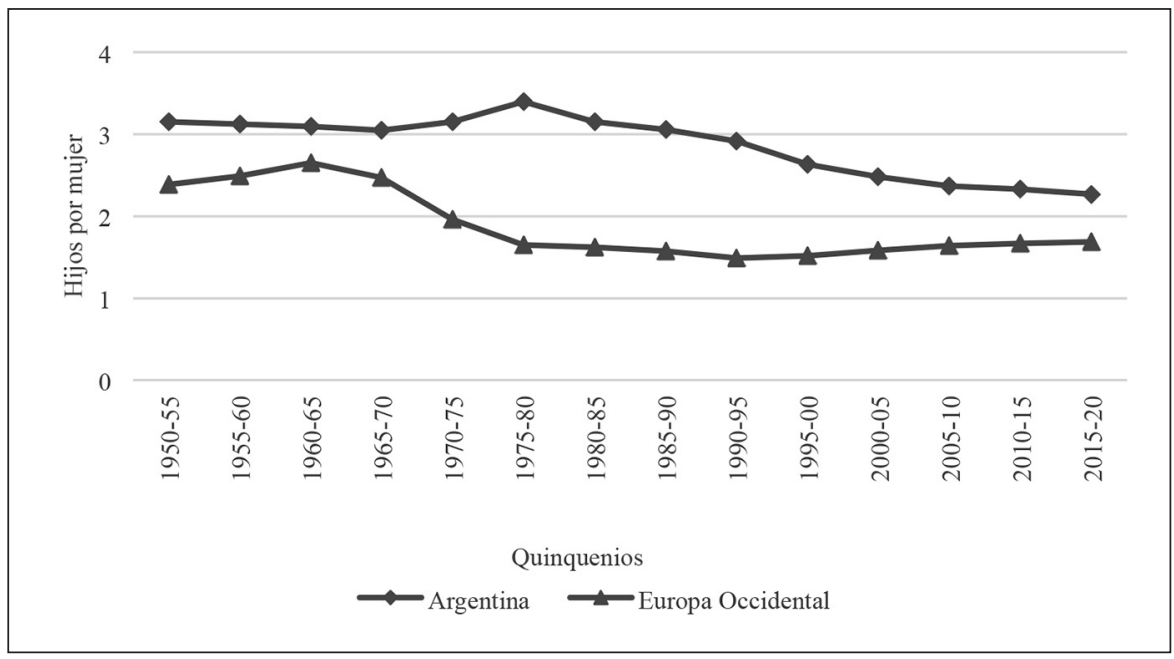

Fuente: Elaboración propia con datos de la División de Población de las Naciones Unidas, World Population Prospect, Revisión 2019. 


\section{GRAFICO 4}

TASAS DE FECUNDIDAD POR GRUPOS DE EDAD, ARGENTINA, 1975-79 Y 2015-2020

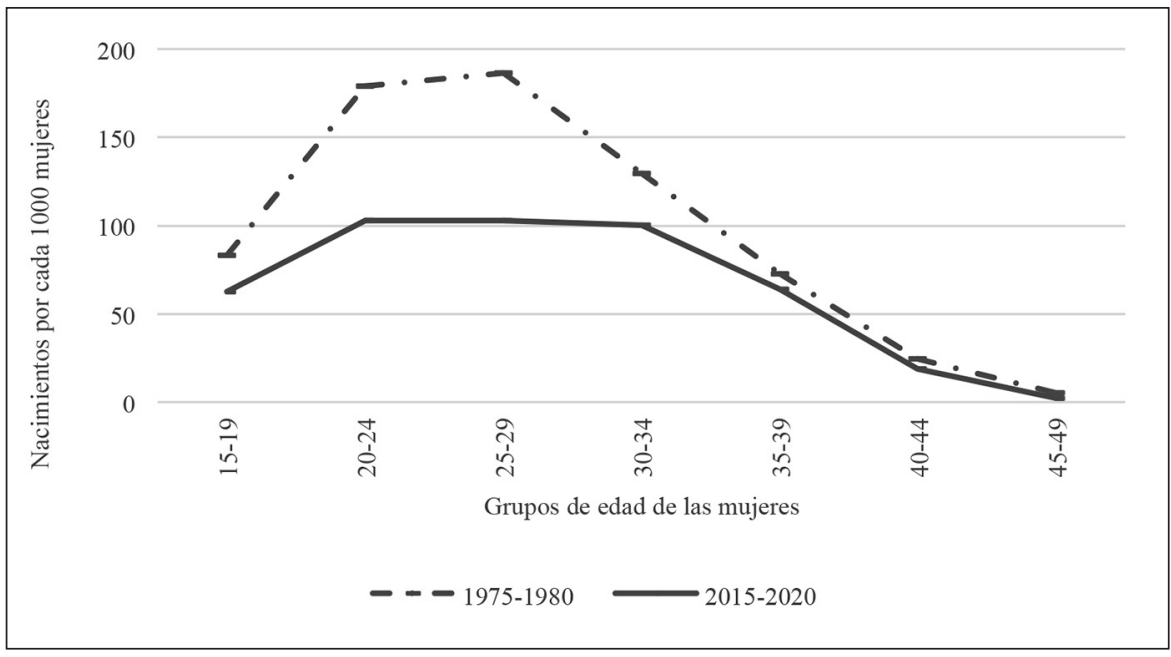

Fuente: Ídem Gráfico 3.

La baja tasa de fecundidad de Argentina en la Región contribuye a ubicar al país entre aquellos con un nivel de envejecimiento relativamente alto en América Latina. Un indicador de este fenómeno es la tasa de soporte, esto es, en una de sus tantas versiones, la cantidad de personas de 20 a 64 años por cada cien de 65 años y más ${ }^{3}$. En el Gráfico 5 se muestra la evolución temporal de esta tasa y la comparación del país con el promedio mundial.

El envejecimiento de la población no implica corresidencia intergeneracional (Paz, 2016), pero es indudable que hay una fuerte relación entre esta y el cambio en la estructura demográfica (Guzmán \& Hakkert, 2001). Lo que aumenta con la prolongación de la esperanza de vida a edades avanzadas es la demanda de cuidado, y en este caso la corresidencia intergeneracional es la manera de captar, aunque de manera indirecta, la demanda de cuidado de la población de personas mayores cuando se dispone de datos más precisos.

Para lograr una aproximación más precisa al problema que se aborda en este artículo conviene utilizar el concepto de "envejecimiento doméstico" (Bertranou, 2008), el que permite relacionar el aumento en la proporción de personas mayores en la población con la corresidencia y permite acoplar ambas ideas a la de participación económica.

3 Este indicador no solo es uno de los muchos posibles para medir el envejecimiento de la población, sino que es una de las versiones de las tantas posibles. Otras toman al grupo 15-64 como referencia. 


\section{GRAFICO 5}

TASAS DE SOPORTE EN LA ARGENTINA Y EL MUNDO, 1950-2020

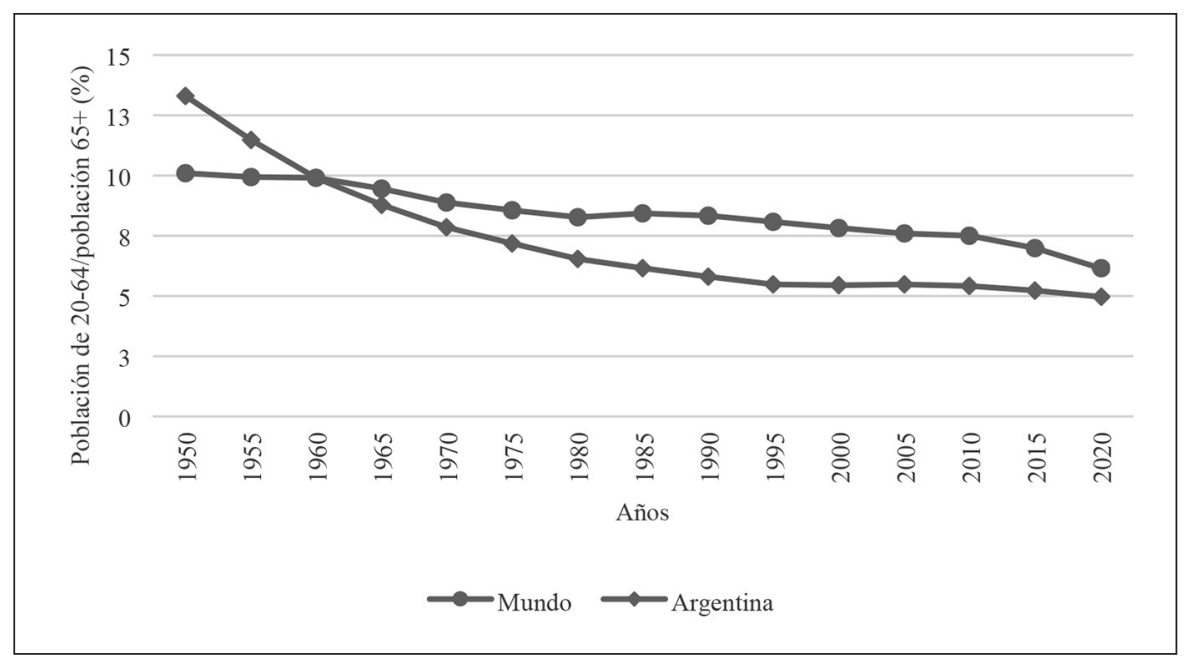

Fuente: Ídem Gráfico 3.

En el Gráfico 6 se muestra uno de los indicadores del envejecimiento doméstico en los hogares argentinos: los hogares en los que hay al menos una persona mayor.

Como lo plantean diversos autores (Bertranou, 2008; Bravo y Puentes, 2012), en países con bajo nivel de desarrollo relativo en sistemas protección formal durante la vejez, la búsqueda de los padres tiene una racionalidad con microfundamento y dispositivos culturales y axiológicos de apoyo. Es el llamado "cuidado informal", que está particularmente extendido en aquellas sociedades donde el sistema formal de cuidados no está lo suficientemente desarrollado o donde, a pesar de existir, no está al alcance de algunos sectores de la población. El tema del cuidado y su relación con la corresidencia permite detectar relaciones entre el proceso de envejecimiento, la demanda de cuidado de la población de personas mayores y la oferta de trabajo de los miembros del hogar en edades centrales. El cuidado, ya sea de niñas y niños y en menor medida de adolescentes y de ancianas y ancianos, es una actividad tiempo intensiva que compite con el número de horas que las personas disponen para el resto de las actividades, como el trabajo y el ocio, entre tantas otras.

La Tabla A.1 del Apéndice contiene los valores medios de las variables para el año 2018, y que son las que se usan en el análisis del resto de los años incluidos en este estudio. Corresponden a dos grupos de adultas/os en edades centrales: hombres y mujeres entre 18 y 54 años de edad y ocupadas/os de ese mismo rango etario. Debido a que interesan solamente los hogares con personas mayores, las muestras 


\section{GRAFICO 6}

TASAS DE ENVEJECIMIENTO DOMESTICO EN ARGENTINA, 2004-2018

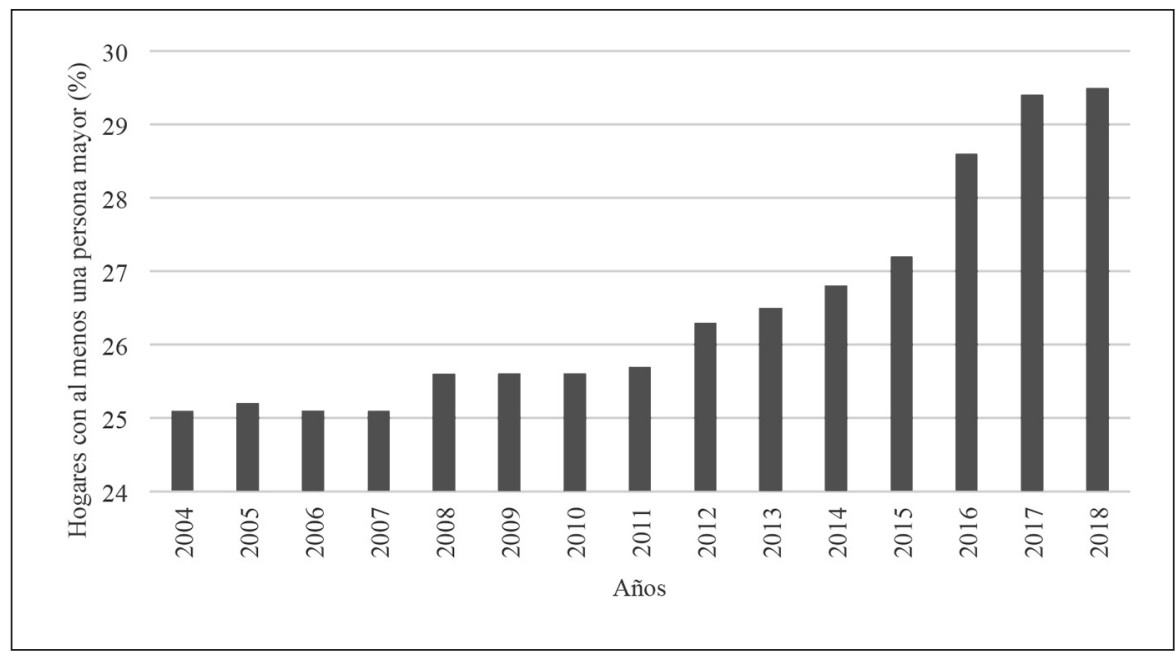

Fuente: Elaboración propia con datos de INDEC, EPH.

finalmente incluidas en las regresiones son más pequeñas que las que contienen al conjunto de adultas/os.

Se destacan como rasgos centrales la mayor tasa de actividad de horas de trabajo de la población masculina. También resulta interesante notar que si bien hay cierta paridad en la cantidad de personas mayores corresidentes, el número de niñas y niños es mayor entre las mujeres que entre los hombres, lo que implica una proporción más alta de hogares monoparentales con jefatura femenina que con jefatura masculina. No se encuentran diferencias importantes de edad entre hombres y mujeres, aunque las/os ocupadas/os son apenas mayores (más o menos en un año) que el conjunto de la población activa. Una diferencia similar se observa para la variable "años de escolaridad", aunque en este caso hay brecha entre hombres y mujeres, favorable a estas últimas.

\section{B. Análisis multivariante}

Con el propósito de evaluar la correlación entre cantidad de personas mayores corresidentes y oferta laboral de hombres y mujeres en edades centrales (18-54), se estimaron los modelos descritos en el apartado B de la sección anterior. Los resultados de esas estimaciones de presentan en las Tablas A.2 y A.3 del Apéndice. En el apartado siguiente se extiende este análisis a otros años del período 2004-2018. 
Se constata que la cantidad de niñas y niños presentes en el hogar se correlaciona positivamente con la participación de los hombres y negativamente con la de las mujeres. Este es un resultado conocido en la literatura y que se ve con las prácticas de cuidado vigentes en muchos países (Killingsworth y Heckman, 1986) ${ }^{4}$. No obstante, si se compara los valores contenidos en la Tabla A.1 con los que provienen de otros estudios pueden existir algunas diferencias debidas a que se están considerando aquí solamente a adultas/os que corresiden con personas mayores y no al conjunto de adultas/os, independiente de la corresidencia intergeneracional.

Centrando la atención en las personas mayores, las dos primeras columnas de la Tabla A.2 (llamada ahí "Versión 1") muestra que no puede rechazarse la hipótesis de ausencia de relación entre el número de personas mayores en los hogares y la participación laboral de hombres y mujeres. Pero al abrir esta variable para observar la estructura por edad y sexo de las personas mayores que corresiden con las/os adultas/os en edades centrales, aparecen situaciones particularmente relevantes.

Primero, la cantidad de mujeres entre 65 a 69 años, y entre 70 a 79 años, está positivamente relacionada con la participación de las mujeres y no tiene correlación con la participación de los hombres. Esta evidencia no permite rechazar la hipótesis de transferencias hacia abajo en términos de cuidado, sugiriendo que las mujeres mayores podrían estar colaborando con las adultas (18-55) en las tareas del hogar, algunas de las cuales pueden estar referidas al cuidado de personas (abuelas cuidadoras).

Segundo, la cantidad de hombres entre 65 y 69 años, y entre 70 a 79 años, está negativamente relacionada con la participación de los hombres en edades centrales y no tiene correlación con la participación femenina. Esto da lugar a suponer que estos hombres mayores, presentes en el hogar, contribuyen con ingresos al sostenimiento del hogar provocando alguna alteración al salario de reserva de los varones en edades centrales.

Tercero, se observa correlación negativa y significativa entre la cantidad de hombres mayores de 69 años y la participación laboral de mujeres en edades centrales. La hipótesis de cuidado toma fuerza en este caso y podría pensarse que son las mujeres en edades centrales las que se hacen cargo de estas personas mayores en el hogar.

Por su parte, la presencia y cantidad de personas mayores no está correlacionada con la cantidad de horas que hombres y mujeres destinan semanalmente al trabajo remunerado (Tabla A.3, Apéndice de Tablas). Los signos y la significancia de las demás variables son similares a los que se obtienen para la participación en la fuerza de trabajo, con excepción de la presencia y cantidad de personas mayores. La cantidad de niñas

4 Montgomery \& Trussell (1986) dicen que se trata de una práctica frecuente la introducción de estas variables en una ecuación de participación. Para estimaciones más recientes se puede ver a Jäntti et al. (2015) y Wu \& Zhou (2015). En las estimaciones se observa que los parámetros para la participación de las mujeres decrecen en valor absoluto conforme aumenta la edad de niñas y niños en el hogar, lo que se adjudica a la mayor demanda de cuidado de las/os más pequeñas/os. Como se ve aquí eso no pasa siempre y en algunos casos no se verifica significatividad estadística. 
y niños en estos hogares está relacionada positivamente con las horas trabajadas por la población masculina entre 18 y 55 años. Es decir, que esta variable impacta tanto en la decisión de participar como en el esfuerzo laboral propiamente dicho, medido por las horas trabajadas.

Las variables de control se comportaron de manera esperada tanto para hombres como para mujeres en edades centrales ${ }^{5}$ : la participación describe un perfil con forma de " $U$ " invertida respecto a la edad, aumenta a medida que crece el nivel educativo de las personas y, salvo algunas excepciones, es más alta en el Gran Buenos Aires que en las demás regiones del país.

\section{Extensión del período}

Con el fin de detectar robustez temporal de los resultados comentados hasta aquí se estimaron los modelos probit y tobit para participación y horas, respectivamente, para el período comprendido entre los años 2004-2018. La de 2004 es la primera base de datos disponible comparable con la actual (año 2018), dado que, como se explicó en el apartado A, sección V, la modalidad continua se consolidó precisamente en este año. El Cuadro 1 resume los resultados de la variable más general: cantidad de personas mayores en el hogar.

Tomando el promedio simple del período puede decirse que la tasa de actividad masculina supera en 19 puntos porcentuales a la femenina y que los hombres trabajan para el mercado 9 horas más que las mujeres. En 17 de las 60 regresiones corridas los parámetros relevantes son significativamente diferentes de cero. De esas 17, más de la mitad corresponde a la tasa de actividad masculina.

Dada esta evidencia puede decirse que la cantidad de personas mayores en el hogar está inversamente relacionada con la tasa de actividad, principalmente masculina. La evidencia que permite sostener correlación entre la presencia y número de personas mayores en el hogar y la participación femenina es más escasa, excepto en lo que hace a las horas trabajadas, variable para la cual los dos parámetros que resultaron significativos fueron positivos.

En los Cuadros $2 \mathrm{a}$ y $2 \mathrm{~b}$ se resumen los resultados que se obtienen al considerar la estructura por edad y sexo de las personas mayores corresidentes. En estos casos los parámetros estimados ascienden a 360 que provienen de 60 regresiones y de seis variables explicativas.

En todos los casos se comprueba lo que se había anunciado al analizar el Cuadro 1: la mayor cantidad de parámetros significativos provienen de la relación entre la tasa de actividad masculina y las personas mayores corresidentes. Los parámetros sugieren correlación negativa.

5 "Esperada" de acuerdo al marco conceptual y a los estudios empíricos sobre el tema y analizados en la revisión de la literatura. 


\section{CUADRO 1}

RESUMEN DE 60 REGRESIONES Y VALORES MEDIOS DE LAS VARIABLES DE OFERTA LABORAL

\begin{tabular}{|c|c|c|c|c|c|c|c|c|}
\hline \multirow{2}{*}{ Año } & \multicolumn{2}{|c|}{$\begin{array}{c}\text { Efecto marginal } \\
\text { (pp) }\end{array}$} & \multicolumn{2}{c|}{$\begin{array}{c}\text { Efecto marginal } \\
\text { (horas) }\end{array}$} & \multicolumn{2}{c|}{$\begin{array}{c}\text { Tasa de Actividad } \\
(\%)\end{array}$} & \multicolumn{2}{c|}{ Horas trabajadas } \\
\cline { 2 - 8 } & Hombre & Mujer & Hombre & Mujer & Hombre & Mujer & Hombre & Mujer \\
\hline 2004 & & & & & 83,8 & 66,1 & 42,5 & 33,2 \\
2005 & & & & 1,4 & 83,3 & 64,2 & 43,3 & 33,5 \\
2006 & $-3,5$ & & $-1,3$ & & 83,5 & 65,5 & 43,4 & 33,8 \\
2007 & $-3,5$ & & & & 83,2 & 63,4 & 43,4 & 34,4 \\
2008 & $-3,9$ & & & & 82,6 & 62,8 & 44,3 & 34,1 \\
2009 & $-2,2$ & & $-1,3$ & & 81,9 & 61,7 & 43,0 & 34,2 \\
2010 & & $-2,8$ & & & 80,0 & 61,5 & 43,0 & 34,1 \\
2011 & $-2,2$ & & & & 81,0 & 61,4 & 42,6 & 34,2 \\
2012 & $-2,5$ & & $-1,1$ & & 80,8 & 62,6 & 42,7 & 33,6 \\
2013 & $-2,6$ & & $-1,0$ & & 79,5 & 61,4 & 42,1 & 33,4 \\
2014 & $-3,5$ & & & \multirow{2}{*}{1,1} & 80,2 & 61,4 & 41,7 & 32,7 \\
2015 & $-4,9$ & $-4,0$ & & & 79,0 & 58,4 & 41,8 & 32,8 \\
2016 & & & & & 79,9 & 60,0 & 41,1 & 32,4 \\
2017 & & & & & 79,0 & 61,8 & 40,3 & 32,2 \\
2018 & & & & & 80,3 & 63,0 & 39,7 & 31,2 \\
\hline
\end{tabular}

Nota: Las celdas vacías indican que el parámetro no es significativamente diferente de cero. Los años que se destacan con el sombreado no contienen información para el año completo, sino de uno de los dos semestres, con lo cual los valores no son estrictamente comparables con el resto.

Fuente: Elaboración propia con datos de INDEC, EPH.

Los valores absolutos más elevados corresponden a las variables que representan personas de 80 años y más, sean hombres (Cuadro 2a) o mujeres (Cuadro 2b). También los parámetros correspondientes a las horas son mayores en valor absolutos para estas variables. Los test de diferencia de media permiten rechazar la hipótesis de igualdad en prácticamente todos los casos. Así, por ejemplo, la sustracción de 5,4 puntos porcentuales de tasa de actividad masculina observado en 2013 para el grupo de 80 años y más, mujeres (Cuadro 2a, Panel 1), sería significativamente mayor que el 3,3 observado para el mismo año para corresidentes entre 65 y 69 años de edad.

Ninguno de los parámetros estimados para la oferta laboral masculina es positivo. En términos conceptuales esto podría interpretarse diciendo que independientemente del sexo y de la edad de las personas mayores, cuando esta se correlaciona con la oferta laboral lo hace reduciéndola, tanto en su dimensión de participación como en las horas semanales dedicadas al trabajo remunerado. 


\section{CUADRO 2A}

RESUMEN DE 60 REGRESIONES Y VALORES MEDIOS DE LAS VARIABLES DE OFERTA LABORAL. MUJERES MAYORES (65+) CORRESIDENTES

\begin{tabular}{|c|c|c|c|c|c|c|}
\hline \multirow{2}{*}{ Años } & \multicolumn{2}{|c|}{$65-69$} & \multicolumn{2}{|c|}{$70-79$} & \multicolumn{2}{|c|}{$80+$} \\
\hline & Hombres & Mujeres & Hombres & Mujeres & Hombres & Mujeres \\
\hline & \multicolumn{6}{|c|}{ Panel 1: Tasa de actividad } \\
\hline $\begin{array}{l}2004 \\
2005 \\
2006\end{array}$ & $-4,3$ & $\begin{array}{l}3,4 \\
4,4\end{array}$ & $\begin{array}{l}-3,0 \\
-3,8\end{array}$ & $\begin{array}{l}3,4 \\
4,6\end{array}$ & $\begin{array}{l}-3,5 \\
-3,4\end{array}$ & \\
\hline 2007 & & & $-3,8$ & & $-4,1$ & $-6,0$ \\
\hline $\begin{array}{l}2008 \\
2009 \\
2010 \\
2011 \\
2012 \\
2013 \\
2014\end{array}$ & $\begin{array}{l}-3,2 \\
-3,7 \\
-3,2 \\
-3,8 \\
-3,3\end{array}$ & $\begin{array}{l}5,6 \\
3,7 \\
4,5\end{array}$ & $\begin{array}{l}-4,1 \\
-3,9 \\
-3,2 \\
-3,3 \\
-4,3 \\
-4,8 \\
-3,4\end{array}$ & 4,9 & $\begin{array}{l}-4,9 \\
-4,9 \\
-4,3 \\
-5,3 \\
-5,4 \\
-5,7\end{array}$ & $\begin{array}{l}-4,0 \\
-5,6\end{array}$ \\
\hline $\begin{array}{l}2015 \\
2016\end{array}$ & $-4,1$ & 5,0 & $\begin{array}{l}-4,3 \\
-3,1\end{array}$ & & $-4,9$ & $-6,0$ \\
\hline 2018 & & 4,7 & & 4,6 & & \\
\hline & \multicolumn{6}{|c|}{ Panel 2: Horas semanales trabajadas } \\
\hline $\begin{array}{l}2004 \\
2005 \\
2006\end{array}$ & $-1,5$ & 2,1 & $-4,5$ & 1,7 & $-2,6$ & $-2,2$ \\
\hline 2007 & & & & 2,0 & & \\
\hline $\begin{array}{l}2008 \\
2012 \\
2013 \\
2014\end{array}$ & $\begin{array}{l}-1,4 \\
-1,7\end{array}$ & 2,4 & $-1,9$ & $\begin{array}{l}1,6 \\
1,4\end{array}$ & & \\
\hline
\end{tabular}

Nota y Fuente: Ídem Cuadro 1.

Con las mujeres los resultados son muy diferentes: el sexo y la edad de las personas mayores corresidentes parece tener importancia para la oferta laboral de las mujeres. Cuando la persona mayor es un hombre, la correlación es siempre (cuando lo es) negativa, tanto para participación como para horas. Cuando la persona mayor es mujer, la edad es lo que importa: para las corresidentes menores de 80 años se correlaciona positivamente con la oferta laboral femenina y para las corresidentes de 80 años y más la correlación es similar que la de los hombres corresidentes: negativa.

En suma, la correlación entre la oferta laboral de hombres y mujeres en edades centrales y la presencia y número de personas mayores en el hogar, si bien es elusiva a las pruebas, no puede decirse que no exista: la evidencia no es contundente pero no 


\section{CUADRO 2B}

RESUMEN DE 60 REGRESIONES Y VALORES MEDIOS DE LAS VARIABLES DE OFERTA LABORAL. HOMBRES MAYORES (65+) CORRESIDENTES

\begin{tabular}{|c|c|c|c|c|c|c|}
\hline \multirow{2}{*}{ Años } & \multicolumn{2}{|c|}{$65-69$} & \multicolumn{2}{|c|}{$70-79$} & \multicolumn{2}{|c|}{$80+$} \\
\hline & Hombres & Mujeres & Hombres & Mujeres & Hombres & Mujeres \\
\hline & \multicolumn{6}{|c|}{ Panel 1: Tasa de actividad } \\
\hline $\begin{array}{l}2004 \\
2005 \\
2006\end{array}$ & & & $-3,7$ & $\begin{array}{l}-3,6 \\
-3,1\end{array}$ & & $\begin{array}{l}-7,4 \\
-6,1 \\
-4,7\end{array}$ \\
\hline 2007 & & & & $-5,8$ & $-7,6$ & $-5,9$ \\
\hline $\begin{array}{l}2008 \\
2009 \\
2010 \\
2011 \\
2012 \\
2013 \\
2014\end{array}$ & $\begin{array}{l}-3,1 \\
-4,2\end{array}$ & & $-4,6$ & $\begin{array}{l}-3,2 \\
-3,6 \\
-3,1\end{array}$ & $\begin{array}{l}-5,4 \\
-8,0 \\
-4,1 \\
-4,8\end{array}$ & $\begin{array}{l}-5,3 \\
-8,2 \\
-6,5\end{array}$ \\
\hline $\begin{array}{l}2015 \\
2016\end{array}$ & & $-7,2$ & $-5,7$ & & $\begin{array}{l}-8,5 \\
-5,4\end{array}$ & $-9,8$ \\
\hline 2018 & & & $-2,5$ & & & $-7,2$ \\
\hline \multirow[b]{2}{*}{2006} & \multicolumn{6}{|c|}{ Panel 2: Horas semanales trabajadas } \\
\hline & & & & & $-2,1$ & $-2,7$ \\
\hline 2007 & & & & & $-2,8$ & $-2,8$ \\
\hline $\begin{array}{l}2008 \\
2009 \\
2012 \\
2013\end{array}$ & & & $\begin{array}{l}-1,3 \\
-1,8 \\
-1,1\end{array}$ & $-1,6$ & $-2,6$ & \\
\hline
\end{tabular}

Nota y Fuente: Ídem Cuadro 1.

siempre fue nula. Además, en aquellos casos en que se encuentra, es consistente con las hipótesis planteadas en el marco conceptual: la presencia de personas mayores implica transferencias generacionales hacia arriba y hacia abajo que pueden alterar las decisiones de participación y horas de trabajo.

\section{Advertencia sobre la posible evolución}

Debe tenerse presente que si bien la corresidencia intergeneracional fue aumentando en los últimos años (Gráfico 6), las transferencias intergeneracionales, principalmente con las que tienen que ver con el cuidado de personas mayores, dependen del estado de salud de esas personas mayores, de la posibilidad de acceso y del desarrollo de los sistemas de atención formales. El Gráfico 7 ofrece información 


\section{GRAFICO 7}

ARGENTINA, 2005 Y 2013. MUJERES MAYORES QUE DECLARAN NO TENER PROBLEMAS DE MOVILIDAD Y DE AUTONOMIA PARA HACER ACTIVIDADES COTIDIANAS, SEGUN GRUPOS DE EDAD

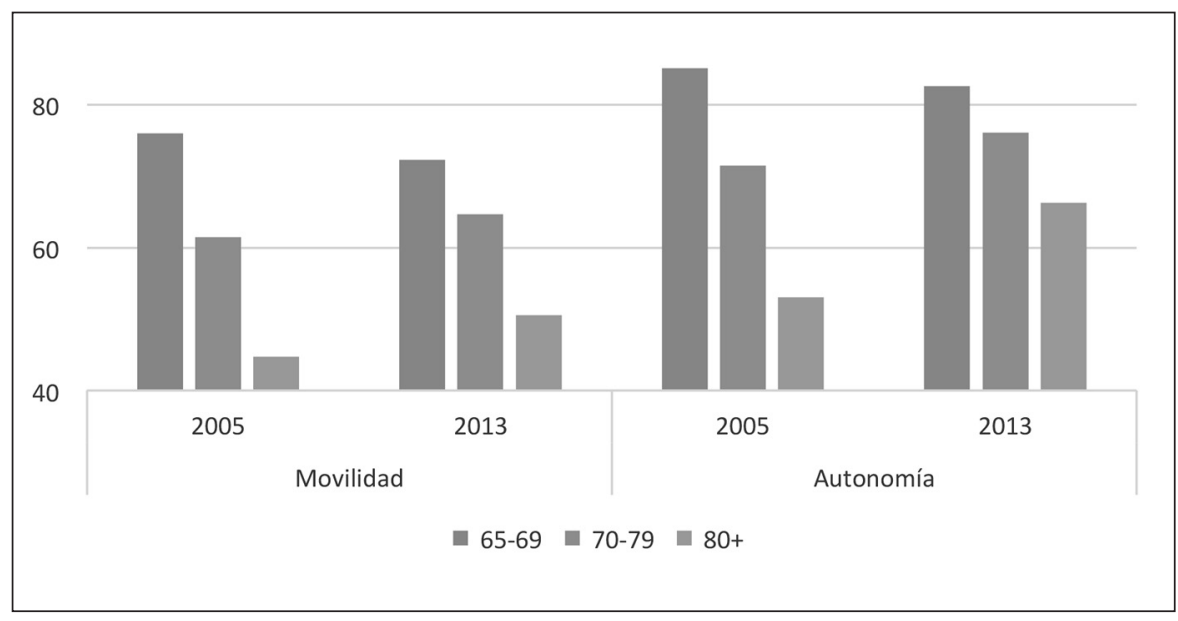

Fuente: Elaboración propia con datos de INDEC-Ministerio de Salud de la Nación. Encuesta Nacional de Factores de Riesgo 2005 y 2013.

que tiene que ver con el primero de esos temas: el estado de salud de las personas mayores. Para su construcción se tuvieron en cuenta solamente personas mayores mujeres, ya que es ese el grupo que estaría negativamente correlacionado con la oferta laboral femenina.

Los datos del Gráfico 7 provienen de dos Encuestas de Factores de Riesgo realizadas en los años 2005 y 2013. Los dos indicadores de salud calculados para mujeres de 70 años y más muestran notables mejorías. Tanto los problemas de movilidad como los de autonomía para realizar actividades cotidianas han avanzado en este período y se cree que en el período que va de 2013 a 2018 puede haber seguido mejorando. Las implicancias de estos cambios debilitan la correlación entre las variables examinadas en este artículo: la participación económica de hombres y mujeres en edades centrales desde la perspectiva de la demanda de cuidado pueden llegar a potenciar la llamada aquí "transferencias intergeneracionales hacia abajo", y que se refieren a las ayudas que estas personas mayores pueden dar a las/os adulta/os en edades centrales liberando tiempo para ser volcado al mercado de trabajo. 


\section{CONSIDERACIONES FINALES}

Se analizó aquí, con datos disponibles de Argentina, la relación entre la cantidad de personas mayores residentes en los hogares y la oferta laboral de hombres y mujeres en edades centrales. Los estudios tradicionales de este tipo suelen considerar el número de hijos como un determinante clave de la participación laboral, pero el número de personas mayores ha sido menos estudiado, a pesar de la importancia creciente de este grupo demográfico ocurrido como consecuencia del aumento de la longevidad y de la caída de la fecundidad observada en todos los países del mundo.

La investigación se concentró en hogares con personas mayores y se evaluaron correlaciones entre la edad y el sexo de las personas mayores corresidentes (como proxies del estado de salud y de las pautas de cuidado y ayuda intrahogar, respectivamente), y dos indicadores de oferta laboral: la participación en la fuerza de trabajo y las horas semanales dedicadas por adultas y adultos en edades centrales a las actividades remuneradas.

Se encontró asociación entre la edad y el sexo de las personas mayores corresidentes y la participación laboral de adultas/os en edades centrales. La asociación de estas variables con respecto a las horas semanales trabajadas fue menos clara, no pudiéndose en muchos casos rechazar la hipótesis de ausencia de relación, la cual podría deberse a una compensación de efectos, como se explicitó oportunamente en el marco conceptual. Por su parte, en aquellos casos en los que se encontró evidencia de relación, la dirección de las mismas responde a lo previsto en dicho marco: hay personas mayores que colaborarían con las tareas domésticas no remuneradas liberando tiempo de adultas en edad central, y otro grupo que demandaría cuidado, reduciendo el tiempo disponible de adultas y adultos en edad central (18-54 años).

Las proyecciones demográficas y sanitarias muestran una tendencia al envejecimiento demográfico y doméstico, a la corresidencia intergeneracional y a la vez una mejora en el estado de salud de las personas mayores. La sí llamada "segunda transición demográfica" con las consecuencias propias de la prolongación de la esperanza de vida y la caída de la fecundidad, podría provocar algunos cambios en los patrones de oferta de trabajo que mitigarían el efecto expansivo provocado por un aumento en la educación de las mujeres y en la reducción del número de hijos por ellas tenidos.

No obstante, se verifica también una clara mejoría en la salud de las personas mayores que acompaña el proceso de prolongación de la longevidad. Esto podría provocar las llamadas aquí transferencias hacia abajo, en el sentido de la ayuda que estas personas pueden prestar a los hogares con adultas y adultos en edades centrales, liberando tiempo disponible y generando las condiciones para una expansión de la oferta de trabajo. 


\section{APENDICE DE TABLAS}

TABLA A. 1

DEFINICION DE LAS VARIABLES USADAS Y VALORES MEDIOS.

ARGENTINA, 2018

\begin{tabular}{|c|c|c|c|c|}
\hline \multirow{2}{*}{ Variables } & \multicolumn{2}{|c|}{ Total $18-54$} & \multicolumn{2}{|c|}{ Ocupadas/os 18-54 } \\
\hline & Hombres & Mujeres & Hombres & Mujeres \\
\hline Actividad/Horas & 0,803 & 0,630 & 39,7 & 31,2 \\
\hline Niñas y niños & & & & \\
\hline $0-4$ & 0,196 & 0,284 & 0,215 & 0,237 \\
\hline $5-12$ & 0,310 & 0,437 & 0,328 & 0,413 \\
\hline $13-17$ & 0,208 & 0,278 & 0,200 & 0,278 \\
\hline Personas mayores mujeres & & & & \\
\hline $65-69$ & 0,262 & 0,246 & 0,271 & 0,256 \\
\hline $70-79$ & 0,320 & 0,288 & 0,328 & 0,311 \\
\hline $80+$ & 0,148 & 0,161 & 0,142 & 0,162 \\
\hline Personas mayores hombres & & & & \\
\hline $65-69$ & 0,202 & 0,218 & 0,195 & 0,203 \\
\hline $70-79$ & 0,211 & 0,208 & 0,213 & 0,209 \\
\hline $80+$ & 0,088 & 0,087 & 0,085 & 0,080 \\
\hline Características personales & & & & \\
\hline Edad & 34,6 & 35,3 & 36,2 & 37,7 \\
\hline Años de escolaridad & 10,9 & 11,9 & 11,2 & 12,8 \\
\hline Región de residencia & & & & \\
\hline Noroeste & 0,280 & 0,290 & 0,286 & 0,286 \\
\hline Nordeste & 0,109 & 0,121 & 0,104 & 0,115 \\
\hline Cuyo & 0,108 & 0,106 & 0,115 & 0,109 \\
\hline Centro & 0,240 & 0,231 & 0,238 & 0,235 \\
\hline Sur & 0,088 & 0,077 & 0,082 & 0,074 \\
\hline Número de observaciones & 8106 & 7445 & 5605 & 3964 \\
\hline
\end{tabular}

Fuente: Elaboración propia, con datos de INDEC-EPH. 
TABLA A. 2

EFECTOS MARGINALES DEL MODELO PROBIT PARA LA PARTICIPACION LABORAL. ARGENTINA, 2018

\begin{tabular}{|c|c|c|c|c|}
\hline \multirow{2}{*}{ Variables explicativas } & \multicolumn{2}{|c|}{ Versión 1} & \multicolumn{2}{|c|}{ Versión 2} \\
\hline & Hombres & Mujeres & Hombres & Mujeres \\
\hline Niñas y niños (0-17) & $0,032 * * *$ & $-0,015 * * *$ & & \\
\hline Personas mayores $(65+)$ & $\begin{array}{l}(0,004) \\
-0,015\end{array}$ & $\begin{array}{c}(0,005) \\
0,009\end{array}$ & & \\
\hline & $(0,010)$ & $(0,014)$ & & \\
\hline Niñas y niños & & & & \\
\hline $0-4$ & & & $\begin{array}{c}0,069 * * * \\
(0,010)\end{array}$ & $\begin{array}{c}-0,030 * * * \\
(0,010)\end{array}$ \\
\hline $5-12$ & & & $0,021 * * *$ & $-0,023 * * *$ \\
\hline 13-17 & & & $\begin{array}{l}(0,007) \\
0,016 *\end{array}$ & $\begin{array}{l}(0,008) \\
0,019 *\end{array}$ \\
\hline $13-17$ & & & $(0,009)$ & $(0,011)$ \\
\hline Personas mayores - Mujeres & & & & \\
\hline 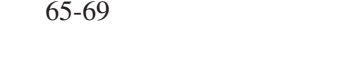 & & & $\begin{array}{l}-0,002 \\
(0,013)\end{array}$ & $\begin{array}{c}0,047 * * \\
(0,018)\end{array}$ \\
\hline $70-79$ & & & $-0,016$ & $0,045^{* *}$ \\
\hline $80+$ & & & $\begin{array}{l}(0,013) \\
-0,019\end{array}$ & $\begin{array}{c}(0,019) \\
0,016\end{array}$ \\
\hline & & & $(0,016)$ & $(0,022)$ \\
\hline Personas mayores - Hombres & & & & \\
\hline $65-69$ & & & $\begin{array}{c}-0,023^{*} \\
(0,014)\end{array}$ & $\begin{array}{c}0,010 \\
(0,020)\end{array}$ \\
\hline $70-79$ & & & $\begin{array}{c}-0,026^{* *} \\
(0,012)\end{array}$ & $\begin{array}{l}-0,009 \\
(0,018)\end{array}$ \\
\hline $80+$ & & & $-0,013$ & $-0,072 * * *$ \\
\hline & & & $(0,016)$ & $(0,025)$ \\
\hline Controles & & & & \\
\hline Edad & $\begin{array}{c}0,068^{* * * *} \\
(0,003)\end{array}$ & $\begin{array}{c}0,089 * * * \\
(0,004)\end{array}$ & $\begin{array}{c}0,066^{* * * *} \\
(0,003)\end{array}$ & $\begin{array}{c}0,087 * * * \\
(0,004)\end{array}$ \\
\hline Cuadrado de la edad & $\begin{array}{c}-0,001 * * * \\
(0,000)\end{array}$ & $\begin{array}{c}-0,001 * * * \\
(0,000)\end{array}$ & $\begin{array}{c}-0,001 * * * \\
(0,000)\end{array}$ & $\begin{array}{c}-0,001 * * * \\
(0,000)\end{array}$ \\
\hline Años de escolaridad & $\begin{array}{c}0,017 * * * \\
(0,001)\end{array}$ & $\begin{array}{c}0,045^{* * *} * \\
(0,002)\end{array}$ & $\begin{array}{c}0,017 * * * \\
(0,001)\end{array}$ & $\begin{array}{c}0,045^{* * *} * \\
(0,002)\end{array}$ \\
\hline Región de residencia & & & & \\
\hline Noroeste & $\begin{array}{c}-0,068^{* * *} * \\
(0,015)\end{array}$ & $\begin{array}{c}-0,111 \text { *** } \\
(0,019)\end{array}$ & $\begin{array}{c}-0,070 * * * \\
(0,015)\end{array}$ & $\begin{array}{c}-0,112 \text { *** } \\
(0,019)\end{array}$ \\
\hline Nordeste & $\begin{array}{c}-0,113 * * * \\
(0,021)\end{array}$ & $\begin{array}{c}-0,173 * * * \\
(0,024)\end{array}$ & $\begin{array}{c}-0,116 * * * \\
(0,021)\end{array}$ & $\begin{array}{c}-0,173 * * * \\
(0,024)\end{array}$ \\
\hline Cuyo & $\begin{array}{c}-0,064 * * * \\
(0,020)\end{array}$ & $\begin{array}{c}-0,113 * * * \\
(0,024)\end{array}$ & $\begin{array}{c}-0,065 * * * \\
(0,020)\end{array}$ & $\begin{array}{c}-0,111 * * * \\
(0,025)\end{array}$ \\
\hline Centro & $\begin{array}{c}-0,031 * * \\
(0,015)\end{array}$ & $\begin{array}{l}-0,031 \\
(0,019)\end{array}$ & $\begin{array}{c}-0,032 * * \\
(0,015)\end{array}$ & $\begin{array}{l}-0,031 \\
(0,019)\end{array}$ \\
\hline Sur & $-0,099 * * *$ & $-0,111 * * *$ & $-0,099 * * *$ & $-0,113 * * *$ \\
\hline & $(0,022)$ & $(0,027)$ & $(0,022)$ & $(0,027)$ \\
\hline Pseudo- $\mathrm{R}^{2}$ & 0,145 & 0,160 & 0,148 & 0,164 \\
\hline Observaciones & 8,106 & 7,445 & 8,106 & 7,445 \\
\hline
\end{tabular}

Nota: Los valores entre paréntesis son desvíos estándar de los que se ignora el signo. Los asteriscos indican significatividad al: $* * * 1 \%, * * 5 \%, * 10 \%$. La ausencia de asterisco implica que no puede rechazarse la hipótesis de ausencia de relación.

Fuente: Elaboración propia con datos de INDEC, EPH. 
TABLA A.3

EFECTOS PARA LAS HORAS DE TRABAJO. ARGENTINA, 2018

\begin{tabular}{|c|c|c|c|c|}
\hline \multirow{2}{*}{ Variables explicativas } & \multicolumn{2}{|c|}{ Versión 1} & \multicolumn{2}{|c|}{ Versión 2} \\
\hline & Hombres & Mujeres & Hombres & Mujeres \\
\hline Niñas y niños (0-17) & $1,012 * * *$ & $-0,141$ & & \\
\hline & $\begin{array}{l}(0,185) \\
-0.273\end{array}$ & $\begin{array}{c}(0,214) \\
0.533\end{array}$ & & \\
\hline Personas mayores $(65+)$ & $\begin{array}{l}-0,273 \\
(0,476)\end{array}$ & $\begin{array}{c}0,533 \\
(0,579)\end{array}$ & & \\
\hline Niñas y niños & & & & \\
\hline $0-4$ & & & $\begin{array}{l}0,996 * * \\
(0,409)\end{array}$ & $\begin{array}{l}-0,267 \\
(0,479)\end{array}$ \\
\hline $5-12$ & & & $1,345 * * *$ & $-0,082$ \\
\hline $13-17$ & & & $\begin{array}{c}(0,328) \\
0,456\end{array}$ & $\begin{array}{l}(0,356) \\
-0,106\end{array}$ \\
\hline & & & $(0,439)$ & $(0,455)$ \\
\hline $\begin{array}{l}\text { Personas mayores - Mujeres } \\
\text { 65-69 }\end{array}$ & & & 0,382 & 0,520 \\
\hline & & & $(0,631)$ & $(0,761)$ \\
\hline $70-79$ & & & $-0,915$ & 1,080 \\
\hline $80+$ & & & $\begin{array}{l}(0,641) \\
-0747\end{array}$ & $\begin{array}{c}(0,775) \\
0.469\end{array}$ \\
\hline & & & $(0,789)$ & $(0,888)$ \\
\hline $\begin{array}{l}\text { Personas mayores - Hombres } \\
\quad 65-69\end{array}$ & & & $-0,077$ & 0,042 \\
\hline 70-79 & & & $-0,440$ & 0,466 \\
\hline $80+$ & & & $\begin{array}{l}(0,599) \\
-0,710\end{array}$ & $\begin{array}{c}(0,720) \\
0,550\end{array}$ \\
\hline & & & $(0,831)$ & $(0,992)$ \\
\hline Controles & & & & \\
\hline Edad & $\begin{array}{c}0,068^{* * * *} \\
(0,003)\end{array}$ & $\begin{array}{c}0,089^{* * *} \\
(0,004)\end{array}$ & $\begin{array}{c}1,011 * * * \\
(0,176)\end{array}$ & $\begin{array}{c}0,682 * * * \\
(0,216)\end{array}$ \\
\hline Cuadrado de la edad & $\begin{array}{c}-0,001 * * * \\
(0,000)\end{array}$ & $\begin{array}{c}-0,001 * * * \\
(0,000)\end{array}$ & $\begin{array}{c}-0,011 * * * \\
(0,002)\end{array}$ & $\begin{array}{c}-0,008 * * * \\
(0,003)\end{array}$ \\
\hline Años de escolaridad & $\begin{array}{c}0,017 * * * * \\
(0,001)\end{array}$ & $\begin{array}{c}0,045^{* * * *} \\
(0,002)\end{array}$ & $\begin{array}{c}-0,126 * * \\
(0,064)\end{array}$ & $\begin{array}{l}0,164 * * \\
(0,077)\end{array}$ \\
\hline Región de residencia & & & & \\
\hline Noroeste & $\begin{array}{c}-0,068 * * * \\
(0,015)\end{array}$ & $\begin{array}{c}-0,111 * * * \\
(0,019)\end{array}$ & $\begin{array}{c}-3,612 * * * \\
(0,636)\end{array}$ & $\begin{array}{l}-4,175 * * * \\
(0,736)\end{array}$ \\
\hline Nordeste & $\begin{array}{c}-0,113 * * * \\
(0,021)\end{array}$ & $\begin{array}{c}-0,173 * * * \\
(0.024)\end{array}$ & $\begin{array}{l}-1,213 \\
(0.818)\end{array}$ & $\begin{array}{l}-1,214 \\
(0.921)\end{array}$ \\
\hline Cuyo & $-0,064 * * *$ & $-0,113 * * *$ & $-1,317^{*}$ & $\begin{array}{l}-1,433 \\
(0,935)\end{array}$ \\
\hline Centro & $-0,031^{* *}$ & $-0,031$ & $-2,782 * * *$ & $-2,518 * * *$ \\
\hline Sur & $-0,099 * * *$ & $-0,111 * * *$ & $-0,746$ & $-1,255$ \\
\hline & $(0,022)$ & $(0,027)$ & $(0,884)$ & $(1,065)$ \\
\hline & $20,314 * * *$ & $17,137 * * *$ & $21,807 * * *$ & $17,704 * * *$ \\
\hline Pseudo-R ${ }^{2}$ & $(3,040)$ & $(3,788)$ & $(3,108)$ & $(3,875)$ \\
\hline Observaciones & 5,605 & 3,964 & 5,605 & 3,964 \\
\hline
\end{tabular}

Nota: Los valores entre paréntesis son desvíos estándar de los que se ignora el signo. Los asteriscos indican significatividad al: $* * * 1 \%, * * 5 \%, * 10 \%$. La ausencia de asterisco implica que no puede rechazarse la hipótesis de ausencia de relación.

Fuente: Elaboración propia con datos de INDEC, EPH. 


\section{REFERENCIAS}

BECCARIA, L.; R. MAURIZIO, R. y G. VAZQUEZ (2017). "El estancamiento de la tasa de participación económica femenina en Argentina en los años 2000”, Desarrollo Económico, vol. 57, N. 221, pp. 8-34.

BERTRANOU, E. (2008). Tendencias demográficas y protección social en América Latina y el Caribe, Centro Latinoamericano de Demografía (CELADE), Comisión Económica para América Latina y el Caribe (CEPAL), Serie Población y Desarrollo Nro. 82, Santiago.

BLAU, F. y L. KAHN (1996). "Wage Structure and Gender Earnings Differentials: An International Comparison" Economica, New Series, Supplement: Economic Policy and Income Distribution, 63(250), pp. S29-S62.

BLOOM, D.; D. CANNING; G. FINK, G. and J. FINLAY (2009). "Fertility, female labor force participation, and the demographic dividend" Journal of Economic Growth, 14, pp. 79-101.

BRAVO, J. y F. PUENTES (2012). Female Labor Force Participation and Informal Care of Adults: Evidence for a Middle-Income Country. Documentos de Trabajo del Departamento de Economía de la Universidad de Chile, Nro. 353, Santiago.

CARMICHAEL, F. (2010). "Who will care? Employment participation and willingness, it supplies informal care” Journal of Health Economics, 29(1), pp. 182-190.

ETTNER, S. (1995). “The impact of 'parent care' on female labor supply decisions", Demography, 32(1), pp. 63-80.

ETTNER, S. (1996). "The Opportunity Costs of Elder Care” Journal of Human Resources, 31(1), pp. 189-205.

GASPARINI, L. and M. MARCHIONNI (Ed.) (2015). Bridging gender gaps? The rise and deceleration of female labor force participation in Latin America. CEDLAS-IDRC, Buenos Aires.

GUZMAN, J. y R. HAKKERT (2001). Envejecimiento demográfico y arreglos residenciales de vida en América Latina. UNFPA, Working Papers Series Nro. 21, México City.

INSTITUTO NACIONAL DE ESTADISTICA Y CENSOS (INDEC) (2013). Proyecciones provinciales de población por sexo y grupos de edad 2010-2040, Serie Análisis Demográfico No 36, Buenos Aires.

HEALTH, R. and S. JAYACHANDRAN (2017). The Causes and Consequences of Increased Female Education and Labor Force Participation in Developing Countries, National Bureau of Economic Research, Working Paper Nro. 493, Washington D. C.

HEITMUELLER, A. (2004). The Chicken or the Egg? Endogeneity in Labour Market Participation of Informal Careers in England. IZA Discussion Papers n ${ }^{\circ} 1366$, October.

JÄNTTI, M. J. PIRTTILÄ and H. SELINE (2015). "Estimating labour supply elasticities based on crosscountry micro data: A bridge between micro and macro estimates?" Journal of Public Economics, 127: pp. 87-99.

KILLINGSWORTH, M. y J. HECKMAN (1986). "Female labor supply: A survey" Chapter 2 in ASHENFELTER, Ch. and R. LAYARD. (Editors): Handbook of Labor Economics, vol. 1: pp. $103-$ 204, Elsevier.

KLASEN, S. and J. PIETERS (2015). What Explains the Stagnation of Female Labor Force Participation in India Urbana? World Bank Policy Research Working Paper Nro. 7222, Washington D. C.

LEE, J. and H. LEE (2016). "Human Capital in the Long Run" Journal of Development Economics, 122 , pp. 147-169.

LEIGH, A. (2010). “Informal Care and Labor Market Participation” Labour Economics, 17(1): pp. 140-149.

LIU, L. (2010). Parental Care Married Women's Labor Time Allocation in Rural China. Mimeo, Institute of Population Research, Peking University.

MAGNANI, E. and A. RAMMOHAM (2006). The effect of Elderly Caregiving on Female Labour Supply in Indonesia. University of New South Wales and University of Sidney.

MAURER-FAZIO, M.; R. CONNELY; Ch. LAN; and L. TANG (2009). Childcare, Eldercare, and Labor Force Participation of Married Women in Urban China:1982-2000. IZA Discussion Paper Nro. 4204, Bonn.

MARTINEZ, C.; T. MILLER, T. y P. SAAD (2013). Participación laboral femenina y bono de género en América Latina. CEPAL-CELADE.IDRC, LC/W.570, Project Documents, Santiago.

MARULL, C. (2011). Gender Inequalities in Time Use in Peru: The determination of market oriented work supply and nonmarket work supply. Dissertation Master Course in Applied Labour Economics for Development, Turin. 
MEDEIROS, M. R. GUERREIRO y J. COSTA (2007). Gender Inequality in Allocating Time to Paid and Unpaid Work: Evidence from Bolivia, The Levy Economics Institute of Band College, Working Paper No. 495.

MENG, A. (2009). Informal home care and labor force Participation of household members. Rhur Economic Papers, Nro. 152.

MONTGOMERY, M. and J. TRUSSELL (1986). "Models of marital status and childbearing" Chapter 3 in ASHENFELTER, Ch. and R. LAYARD. (Editors): Handbook of Labor Economics, vol. 1: 205271, Elsevier.

PAGES, C. y C. PIRAS (2010). El dividendo de género. Cómo capitalizar el trabajo de las mujeres. Banco Interamericano de Desarrollo, Washington D. C.

PAZ, J. (2018). "Brecha de participación económica entre hombres y mujeres y dividendo de género: factores determinantes no tradicionales captados en una muestra de países" Notas de Población, XLV (107): 71-101.

PAZ, J. (2016). "Coresidencia intergeneracional y participación económica de la población en la Argentina, 1970-2010", Desarrollo Económico, Vol. 56, No. 219, pp. 277-307.

PAZ, J. (2009). "El Efecto del Trabajador Adicional. Evidencia para Argentina (2003-2007)" Cuadernos de Economía, 46: 225-241.

PIETERS, J. y S. KLASEN (2011). Drivers of female labour force participation in urban India during India's Economic Boom, Proceedings of the German Development Economics Conference, Berlin 65, Varein für Socialpolitik, Research Committee Development Economics.

POSADAS, J. y VIDAL-FERNANDEZ, M. (2012). Grandparent's Childcare and Female Labor Force Participation. IZA Discussion Papers No. 6398, February.

PSACHAROPOULOS, G. and Z. TZANNATOS (1989). "Female labor force participation: An international perspective" The World Bank Research Observer, 4(2): 187-201.

SHEN, K. and H. CHEN (2012). Does Intergenerational Co-Residence Promote Female Labor Participation: Evidence based on Eastern Province in China. Population Association of America Annual Meeting, Poster Session 3, San Francisco, CA.

SUGAWARA, SH. and J. NAKAMURA (2014). Can Formal Elderly Care Stimulate Female Labor Supply? That Japanese Experience. CIRJE Discussion Papers, Tokyo.

UNGER, M. (2013). Effects of Informal Parental Care on Labor Force Participation in the Nordic Countries. Department of Economics, University of Oslo, May.

VAN HOUTVEN, C.; N. COE; and M. SKIRA (2013). "The effect of informal care on work and wage" Journal of Health Economics, 32(1): 240-252.

WOLF, D. y J. SOLDO (1994). "Married women's allocation of time to employment and care of elderly parents" Journal of Human Resources, 29(4): 1259-1276.

WU, Y. and D. ZHOU (2015). "Women's Labor Force Participation in Urban China, 1990-2010" Chinese Sociological Review, 47:4, 314-342. 